\title{
An Industry 4.0-enabled Low Cost Predictive Maintenance Approach for SMEs:
}

\author{
A Use Case Applied to a CNC Turning Centre
}

\author{
Erim Sezer, David Romero, \\ Federico Guedea \\ Tecnológico de Monterrey, Mexico \\ erimsezer@gmail.com, \\ david.romero.diaz@gmail.com, \\ fguedea@itesm.mx
}

\author{
Marco Macchi \\ Politecnico di Milano, Italy \\ marco.macchi@polimi.it
}

\author{
Christos Emmanouilidis \\ Cranfield University, UK \\ christosem@cranfield.ac.uk
}

\begin{abstract}
This paper outlines the base concepts, materials and methods used to develop an Industry 4.0 architecture focused on predictive maintenance, while relying on low-cost principles to be affordable by Small Manufacturing Enterprises. The result of this research work was a low-cost, easy-to-develop cyber-physical system architecture that measures the temperature and vibration variables of a machining process in a Haas CNC turning centre, while storing such data in the cloud where Recursive Partitioning and Regression Tree model technique is run for predicting the rejection of machined parts based on a quality threshold. Machining quality is predicted based on temperature and/or vibration machining data and evaluated against average surface roughness of each machined part, demonstrating promising predictive accuracy.
\end{abstract}

Keywords-e-Maintenance, Predictive Maintenance, Condition Based Maintenance, Industry 4.0, Smart Manufacturing, Machine Learning, Small Manufacturing Enterprise, Low Cost, Open Source

\section{INTRODUCTION}

In recent years, maintenance activities have attained critical importance for manufacturing enterprises, especially due to the growth in complexity of the interactions between different production activities in increasingly extended manufacturing ecosystems. Meanwhile, the maintenance management discipline has been focusing on the role of maintenance to contribute to delivering business value [1]. The emerging perspective was that maintenance should not be viewed only at a narrow operational and technical level representing an unavoidable cost; but it could be viewed in a long-term strategic view. This strategic role of maintenance can therefore be viewed from the overall business viewpoint, and assessing effectiveness as a main maintenance goal [2-5]. Today, maintenance is clearly seen as a source of added-value, with the key role for driving performance improvement [1].

The increasing numbers of connected machines, systems, equipment and goods on the shop-floor is providing new opportunities as traditional production systems are evolving into so called Cyber-Physical Production Systems (CPPSs), and machine tools into 'smart machining centres' [6] [7]. Maintenance can take advantage to develop Smart Maintenance concepts and applications. Indeed, maintenance engineering is required to this end, as it is considered, traditionally, a lever also to take advantage of new systems and technologies. Certainly, the main goal of maintenance engineering function is to provide high-levels of availability of production equipment by ensuring its maintainability and reliability in order to support production objectives [6]. New systems and technologies are intended to enable maintenance bringing such an added-value through highlevels of performance targets. In particular, ICT for maintenance - in the past, mainly acknowledged through the e-maintenance concept - has especially been developed to bring value through technological innovation for maintenance management [8].

Considering the advances and convergence of Information and Operational Technologies (ITs/OTs) at the Industrial Internet of Things (IIoT), traditional maintenance activities are changing through a transformative paradigmatic shift, having its roots in the e-maintenance concept [9] and now further evolving into smart maintenance. Therefore, in the most recent years, this evolution has been driven by the cognitive computing progress in which predictive maintenance strategies are now fostered by Artificial Intelligent (AI) systems that provide real-time condition monitoring and prognostic capabilities [10]. As a consequence, (smart) industries desire to improve their equipment availability while decreasing downtimes and increasing productivity through e-maintenance / smart maintenance strategies, highly grown with the new era of Industry 4.0. This growth is worth since maintenance costs can represent $15 \%$ to $40 \%$ of the overall costs of goods production [9] [11].

The move from "fail-and-fix" approaches to "predict-andprevent" or proactively plan strategies, was greatly facilitated by the emergence of $e$-maintenance. Today, e-maintenance is further enabled, and transformed, by the use of smart manufacturing technologies [12] such as Industrial Internet of Things (IIoT), cloud-based infrastructure, and wireless communication technologies [9] [13]-[15]. The introduction of such technology enablers further supports the implementation of Condition-Based Maintenance (CBM) strategies, which reduce the need for unnecessary scheduled preventive operations [16][18]. Hence, CBM is based on the monitoring of performance and operation conditions of machine components in order to provide maintenance activities, which can be carried out before a component fails [19] [20].

Research initiatives on e-maintenance have proposed architectures and frameworks which employed web-based information processing, as well as smart sensing and identification to deliver CBM solutions [21]. The added potential of Industry 4.0 technologies further advances the 
technology potential to make CBM approaches feasible for wider range of application settings. Within such architectures/ frameworks, the main idea of CBM is to detect the degradation level of machine components by collecting periodic or continuous real-time data with sensor technologies whereas. CBM strategies may involve Predictive Maintenance (PdM), wherein the current state of monitored equipment, along with historical data and relevant domain knowledge and models is employed to predict through statistical or machine learning models trends, behaviour patterns and correlations [11] [22] [23], and anticipate pending failures in advance to enhance the decision-making process for the maintenance activity [24][26]. PdM is becoming more and more a crucial approach among modern smart manufacturing industries, since global competitiveness is becoming everyday more challenging. Moreover, businesses efficiency benefits from PdM by reducing the lifecycle management costs of production assets, as well as by reducing their downtime. PdM is substantially supported by key Industry 4.0 technologies, such as internet of things and data analytics and is therefore a fundamental part of the Industry 4.0 / Smart Manufacturing landscape.

Machine Learning (ML) methods have been applied for many years now for predictive maintenance systems in different industrial areas. For instance, [20] developed a PdM framework to predict flank wear by using a back propagation neural network, and [15] created a predictive model to forecast failure of a heating, ventilating and air conditioning system using Artificial Neural Networks and Linear Regression. However, the data and services integration opportunities offered in connected production environments make PdM a much more potent approach, capable of exploiting data and knowledge at a breadth and depth that was simply infeasible in the past. The objective of this paper is to present the concept of Industry 4.0 - enabled predictive maintenance and demonstrate it on a machining monitoring application case study.

This paper is organized as follow: Section II reviews the base concepts related to Industry 4.0 technologies and PdM in the context of this research work, Section III details the materials, methods and experimental setup used for developing a low cost PdM strategy based on an Industry 4.0 architecture for SMEs, particularly small manufacturing enterprises, Section IV presents the experimentation results conducted in a CNC turning centre, lastly, Section V summarizes the results of this work and draws conclusions.

\section{BASE CONCEPTS}

Industry 4.0 is the transformation of today's factories into smart factories by using the smart manufacturing technologies such as the Industrial Internet of Things (IIoT), Cyber-Physical Systems (CPSs), Cloud Computing, and Machine Learning (ML) [6] to provide their production systems with flexibility and adaptability capabilities, since present production planning and control approaches are rigid due to the usage of traditional automation and control concepts. Therefore, smart devices such as CPSs, which monitor the production processes of the factory, which are embedded in an IIoT environment, provide a modular and flexible factory structure as well as decentralized decisionmaking support. The application of an Industry 4.0 architecture will help to overcome current production management challenges such as short product lifecycles, high-customized products and stiff global competition [28].

In the reminder, a selected set of base concepts is shortly discussed, considering both the new systems and technologies proving new potential (Section from A to D) and the application target of such potential (Section from $\mathrm{E}$ to $\mathrm{F}$ ).

\section{A. Industrial Internet of Things (IIoT)}

The IIoT uses the Internet of Things (IoT) technologies in an industrial environment such as the manufacturing sector, incorporating machine learning and big data technologies to strengthen the philosophy that "smart machines" show higher efficiency in comparison to humans in terms of accuracy and consistency for data management.

The IIoT connects sensors to machines in order to improve their performance, safety, reliability and energy-efficiency by collecting data from sensors (things) in a cost-efficient way for its strategic interpretation with big data analytic tools that turn the data into actionable information to be delivered the right person and/or computer system at the right time to contribute to the continuous performance improvement of a production system [29].

\section{B. Cyber-Physical Systems (CPSs)}

CPSs are integrated into computers, networks and physical processes to monitor and control the (production) processes with feedback-loops. CPSs are a new generation of systems with computational and physical abilities, including human interaction. The structure of CPSs can be described as a $5 \mathrm{C}$ architecture that consist of five levels: (1) smart Connection, (2) data-to-information Conversion, (3) Cyber, (4) Cognition and (5) Configuration [30].

\section{Cloud Computing}

Cloud computing is a crucial element for the continuous development of the Fourth Industrial Revolution. Simply, cloud computing is the storage and access to data, programs and other virtual resources over the internet (e.g. Application-, Platform-, Infrastructure-as-a-Service). Cloud computing provides various benefits to manufacturing enterprises, specially SMEs, such as: avoid up-front ICT-infrastructure costs (e.g. servers), focus on core-business instead of spending time and money on computer infrastructure, higher applications' speed and agility, improved manageability, less maintenance and rapid adjustment of ICT resources [31].

\section{Machine Learning (ML)}

$M L$ provides the ability to computers to learn without being explicitly programmed. ML is a tool of artificial intelligence, which focuses on the development of computer programs that can change when new data is introduced. ML shows similarities to data mining in which both search for patterns through the data, however, ML detects the pattern through the data and adjusts the program accordingly. Consequently, due to the massive increase of data, manufacturing enterprises changed their decision-making processes in areas such as maintenance, scheduling and quality control. The successful introduction and implementation of ML into the maintenance processes has promised to transform the traditional maintenance approaches to predictive maintenance approaches to support the decision-making processes [32] [33]. 
ML can be mainly divided into (a) supervised-learning and (b) unsupervised-learning. Supervised-learning is when an input variable $(\mathrm{X})$ and an output variable $(\mathrm{Y})$ is given in which the system learns to map inputs and outputs to predict $(\mathrm{Y})$ when new data $(\mathrm{X})$ is inserted into the function. Contrary, unsupervisedlearning takes places if only input data $(\mathrm{X})$ is given and the output is unknown in which the system gets knowledge about the data and discovers the structure and the distribution of the data.

\section{E. Condition-Based Maintenance (CBM)}

$C B M$ is a maintenance strategy that recommends maintenance activities on the basis of an assessment of current machinery condition, inferred through condition monitoring. CBM follows the idea that it is more efficient to determine preventive actions on the basis of current conditions, so as to avoid consequences of any failure of assets, to avoid breakdowns of machines and to reduce maintenance costs by eliminating unnecessary scheduled preventive operations [16] [18] [19] [34] [35]. In early years, maintenance discipline consisted of reacting-only by mechanical breakdowns, which resulted inefficiently for productivity aims. Consequently, as smart manufacturing technology progressed over the time, maintenance activities can increasingly follow a CBM approach.

Today, effective and intelligent CBM systems are expected to detect early forms of degradation, so maintenance costs caused by inaccurate or poor maintenance scheduling can get reduced [36]. Therefore, the practice of PdM strategies can result in an increase in global competitiveness through the elimination of unplanned maintenance operations, since PdM techniques help to identify and to determine the conditions of assets before they fail [22]. Further, PdM helps to monitor the condition of selected physical parameters within an operating machine by performing periodic or continuous real-time data collection [22] [23]. Thus, the collected data can be used to discover, with the help of PdM models, current or future deterioration of components and machines [25] [37].

\section{MATERIALS, Methods \& EXPERIMENTAL SETUP}

This section details the materials, methods and experimental setup used for developing a low cost PdM strategy based on an Industry 4.0 architecture for SMEs, particularly small manufacturing enterprises.

The experimental setup (or architecture) includes various steps to follow, such as: (a) production simulation, (b) quality management, (c) data acquisitions, (d) data storage, and (e) data analytics, in which each step requires various methods and tools to develop a PdM system - for a small manufacturing enterprise. Figure 1 shows the general structure of the proposed Industry 4.0 architecture for the PdM system of a CNC turning centre, using open-source and low-cost technologies.

This paper presents a PdM system based on a simulated production process on a Haas ST $10 \mathrm{CNC}$ turning centre. The CNC machine was setup to produce simple metal parts using a tool holder and tool insert. As for materials, a non-alloy cementing steel with a diameter of 1 inch and a length of 7.87 inch was used for the experimentation. Additionally, a quality control was conducted after each machined part in which the following roughness variables: roughness average $(\mathrm{Ra})$, root mean square rough-ness $(\mathrm{Rq})$ and mean roughness depth $(\mathrm{Rz})$ were manually measured by using a surface roughness tester.

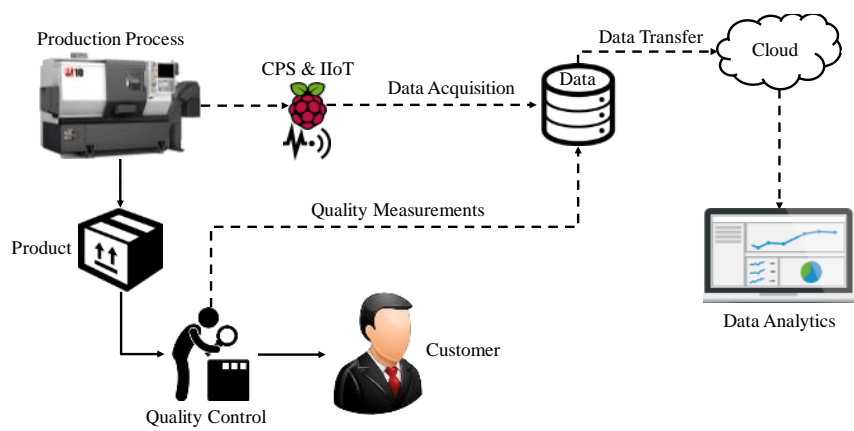

Fig. 1. Schematic Diagram of the Experimental Setup for an Industry 4.0enabled Low Cost PdM Approach for a CNC Turning Centre

\section{A. CPS - Raspberry Pi 3 Model B}

The Raspberry Pi 3 is a low cost third generation singleboard computer. It is employed to offer the multi-functionality of data acquisition, data processing and connectivity for data transmission

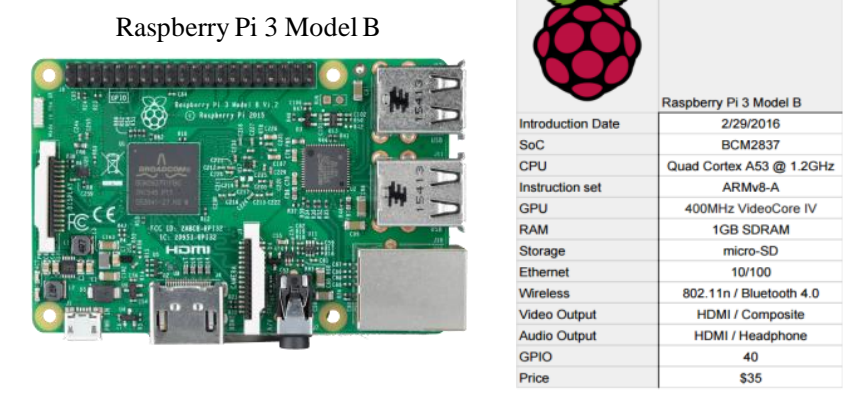

Fig. 2. Raspberry Pi 3 Model B

\section{B. IIoT-Sense HAT}

The add-on board "Sense-HAT" selected for the Raspberry Pi 3 Model B includes various sensors such as gyroscope, accelerometer, magnetometer, temperature, barometric pressure and humidity. The selected Sense-HAT can sense a wide variety of conditions and provide output via the built-in LED matrix (see Figure 3). Its configuration and the access to its sensors is performed by using Python high-level programming language.

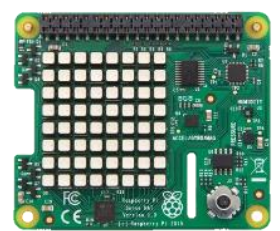

Fig. 3. Raspberry Sense-Hat

\section{Data Management and Processing}

While any relevant cloud infrastructure can be employed in such a context, here Dropbox is simply employed as personal cloud storage service. The main purpose of the selection of Dropbox is due to the easy and fast connection to the Raspberry Pi platform. More specifically, a Dropbox uploader can be installed and configured on the Raspberry Pi 3 Model B via a Python script to provide automatized data exchange and storage. 
One of the most attractive and common tools to develop predictive models is the open-source programming language $\mathrm{R}$. A wide variety of statistical (e.g. linear and nonlinear modelling, classical statistical tests, time-series analysis, classification, clustering, etc.) and graphical techniques are supported in R. The choice of the open-source $\mathrm{R}$ programming language is consistent with the main idea of a low-cost and flexible solution, it can perform operations on entire arrays of numbers without writing loops and it is attractive in terms of package ecosystem.

\section{EXPERIMENTATION}

The experimentation for the above described use case was performed at Haas ST $10 \mathrm{CNC}$ turning centre, and was divided into four main stages: (1) production simulation, (2) data acquisition and storage, (3) PdM framework and (4) results.

\section{A. Stage 1-Production Simulation}

The first stage in the proposed data process chain is about the simulation of a real-life production environment, which requires the selection of material, tool-holder, tool-insert and the development of a machining process. A G-Code for the workpiece, shown in Figure 4, was designed and developed. Position 1 in the work-piece is described as the critical point in which the quality needs to be guaranteed.

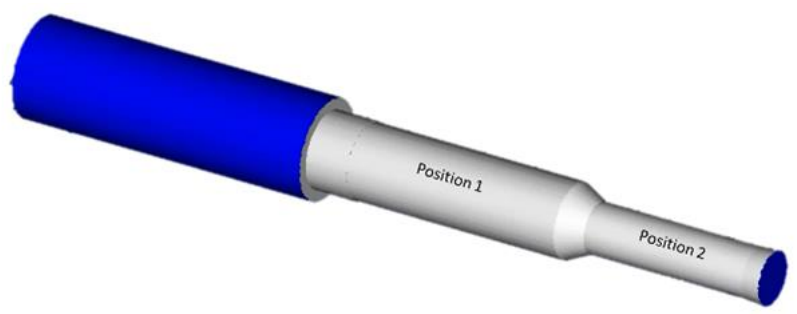

Fig. 4. Machined Work-piece

The experiment is divided into three sets in which the main purpose is to produce work-pieces with different machining parameters (i.e. spindle speed and feed rate) until the insert is worn out or even broken. Table 1 shows three sets of selected machining parameters for the machining process.

\begin{tabular}{|c|c|c|c|}
\hline & $\begin{array}{c}\text { Experiment } \\
\text { ONE }\end{array}$ & $\begin{array}{c}\text { Experiment } \\
\text { TWO }\end{array}$ & $\begin{array}{c}\text { Experiment } \\
\text { THREE }\end{array}$ \\
\hline $\begin{array}{c}\text { Spindle speed } \\
\text { (V) }\end{array}$ & 3600 & 2600 & 1600 \\
\hline $\begin{array}{c}\text { Feed rate } \\
\text { (f) }\end{array}$ & 0.3 & 0.2 & 0.1 \\
\hline $\begin{array}{c}\text { Depth of cut } \\
\text { (d) }\end{array}$ & $1.954 \mathrm{~mm}$ & $1.954 \mathrm{~mm}$ & $1.954 \mathrm{~mm}$ \\
\hline $\begin{array}{c}\text { Cycle time per } \\
\text { Work-piece }\end{array}$ & 80 seconds & 113 seconds & 210 seconds \\
\hline
\end{tabular}

\section{Experiment $O N E$}

With the machining parameters of the Experiment ONE, the cutting tool could produce in total 21 work-pieces until it worn out. Figure 5 shows the behaviour of the measured roughness variables of position " 1 " in which after the 16th piece the tool wear started to accelerate, impacting on the product quality.

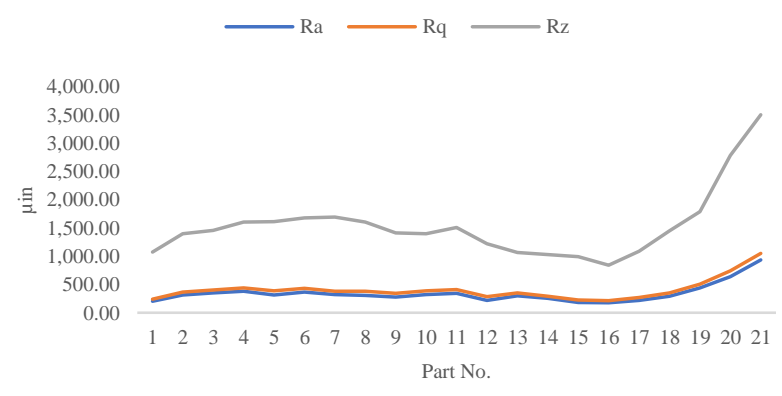

Fig. 5. Roughness Variables Behaviour in Experiment ONE

\section{Experiment TWO}

Furthermore, in Experiment TWO, due to the decreased spindle speed and feed rate, the process could machine 32 workpieces in total until the cutting tool broke. Figure 5 shows, after the 27th piece the surface roughness started to increase significantly and consequently the tool wear started to accelerate.

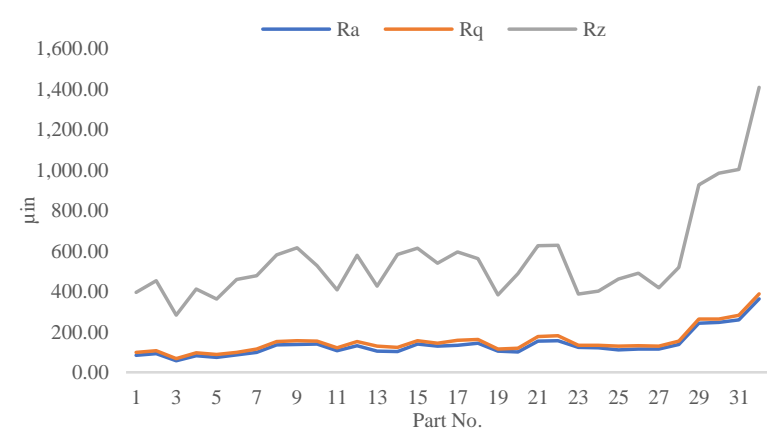

Fig. 6. Roughness Variables Behaviour in Experiment TWO

\section{Experiment THREE}

Finally, due to the decreased spindle speed and feed rate the process could machine 52 work-pieces in total until the cutting tool broke. Figure 7 shows, after the 48th piece the surface roughness started to increase significantly and consequently the tool started to accelerate.

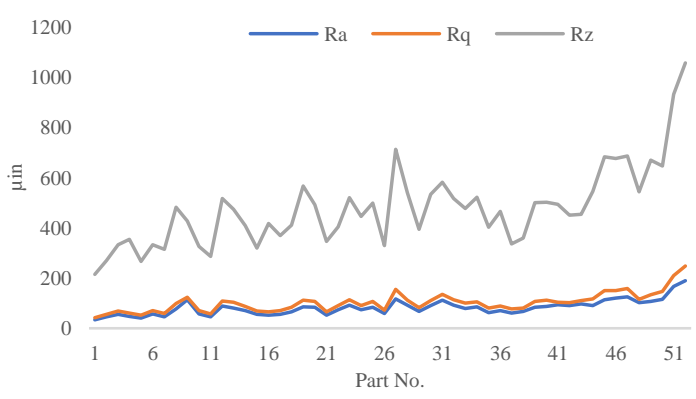

Fig. 7. Roughness Variables Behaviour in Experiment THREE

\section{B. Stage 2-Data Acquisition and Storage}

Before the production process started, a Raspberry Pi 3 Model B with a Sense-Hat was installed into the Haas ST 10 Turning Centre to collect temperature and vibration data. The Sense-Hat chip is connected to the Raspberry Pi via its available pins. As well, a black case is used to cover and protect the device. Figure 8 shows the assembled Raspberry Pi 3 Model 
$B$ with the Sense-Hat, and the case attached to the tool magazine of the CNC machine. The device software runs on an Ubuntu OS, which allows easy programming in Python for the SenseHat and other applications. Once, the Raspberry Pi was setup, the temperature and accelerometer of the Sense-Hat needed to be activated and accessed via Python. For teether free communication between the Raspberry $\mathrm{Pi}$ and an external computer, PuTTY is used, which is a free and open-source application that can be used to connect to serial ports and Secure Shell (SHH) to Raspberry Pi. PuTTY allows fast and easy connection to the Raspberry Pi over SSH and the IP address of the Pi.

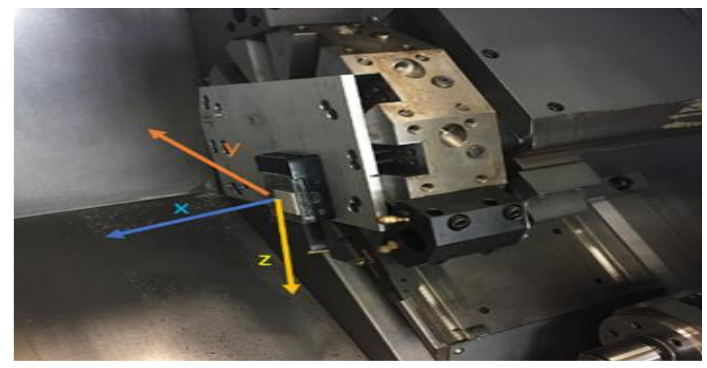

Fig. 8. Haas ST 10 Turning Centre with the CPS Installed

A Dropbox BASH script uploader is employed for handling part of the cloud-based data management. The selection of the BASH script has two main advantages: it is portable, which means it needs only URL and it has security provisions, which means it is not required to provide username or password because it uses the official Dropbox API v2 for the authentication process. Finally, a second Python script was developed for an automatized file transfer from the Raspberry Pi to Dropbox.

\section{Stage 3-PdM Framework}

Once the data was collected and stored successfully in the Cloud, the learning part of the PdM framework was implemented R. Specifically the Recursive Partitioning and Regression Tree (rpart) technique was used. In general, "Decision Trees" can be used for classification or regression problems in which the model is presented as a tree structure. A decision tree can be incrementally built while the dataset is divided into smaller subsets. Furthermore, the result of a decision tree is represented with decision nodes and leaf nodes in which a decision node can have one or more branches and the leaf nodes show decisions on the numerical or categorical target. The root node on the top-most corresponds to the best predictor. The main goal is to create a model that predicts the value of a target variable by learning simple decision rules inferred from the data features. On the other hand, recursive partitioning is a statistical method for multivariable analysis in which a decision tree is created that can classify members of a population precisely by dividing it into subsets. It is called recursive because each subset can be divided into an infinite number of times until the dividing process finishes after certain criteria is reached. The tree is built by two main steps: (a) the single variable is found which best splits the data into two groups, (b) the data is separated, in which the separation process is applied separately to each sub-group recursively until the subgroups either reach a minimum size or until no improvement can be made. The development of the rpart required eight steps in total.
(1) Retrieving raw data from Dropbox into RStudio.

The first step is to retrieve the data from Dropbox and run it into RStudio in order to start the data analysis procedure and the development of the predictive model. The function read.csv () is used to read the data and to store it into the global environment of RStudio.

\section{(2) Performing basic statistical calculations.}

The second step is to write a function, which performs basic statistical calculations such as mean, standard deviation, maximum, mean difference and max difference.

These statistical calculations are used to gain a basic understanding of the behaviour of the data, to feed the decision tree model and to develop a uniform data frame.

(3) Cleaning data and creating data frame for the modelling.

The third step involves the adjustment of the measured data with the roughness variable $\mathrm{Ra}$ since each run of the experimentation collected a large number of data points whereas the roughness variable has only one data point for each run of experimentation. Therefore, for each run the mean values were considered to have the same number of data points for each run and for the roughness variable. More specifically, a uniform data frame is developed.

\section{(4) Normalization of the data.}

The fourth step is about the normalization of the data since the data points for temperature and for acceleration $(\mathrm{x}, \mathrm{y}$ and $\mathrm{z})$ are showing significant differences e.g. a temperature data point might be 22 and a data point for acceleration for $\mathrm{x}$ axis might be -0.987. Therefore, to avoid the differences between the data points, the scale () function is used to determine standardized values for each element in the data-set. Simply, each element of the data-set is set between zero and 1 .

(5) Setting a threshold for the roughness variable (Ra).

Since the experimentations are performed under different machining conditions (rpm and feed rate) the quality threshold for each experiment differs from each other e.g. for Experiment (THREE) a quality threshold for Ra of $30 \mu$ inch is set and for Experiment (ONE) and (TWO) a value of $100 \mu \mathrm{inch}$.

(6) Splitting the data into training and test data-sets.

Splitting the data-set into training and test data-set is essential in predictive modelling. Consequently, the decision tree model is based on the data-set of Experiment (TWO), which is the training set and Experiment (ONE) and (THREE) are used as test sets to perform the predictions. In fact, a training set is a set of data used to discover potential predictive relationships whereas a test set is a set of data used to assess the strength and utility of a predictive relationship.

(7) Initializing the decision tree model.

Once the data is setup, the decision tree model is initialized by applying the rpart () function. As mentioned above, the dataset and the quality threshold from Experiment (TWO) is used to train the recursive partitioning and regression tree. As well, the mean and standard deviation values of temperature and $\mathrm{x}$-axis are considered as predictor variables and the violate variable 
which is a binary value (1) if Ra exceeds threshold and (zero) if $\mathrm{Ra}$ is below the threshold) is used to train the model.

(8) Performing predictions.

The final step involves the prediction process based on the developed decision tree model and the test data (Experiment (ONE) and (THREE)). The function predict () is used in which two arguments are given into the function, namely, the decision tree model and the test data. It is important to mention that the input variables are continuous variables and the output variables categorical, in this case (1) and zero, thus the results of the predictions can be seen as probabilities.

\section{Stage 4-Results}

The key result is the demonstration of the concept of Industry 4.0-enabled monitoring through low cost devices. While the main supported functionality of the presented case support the key constituents of a condition monitoring data process chain, from data acquisition and signal pre-processing, to detection, diagnosis, and prediction, the prime focus of this study has not been to develop a new approach for such data processing, but showcase that such processing can be delivered through low-cost IoT-enabled simple architecture. Furthermore, we present some indicative findings on the processing capabilities for the experimental setup, again as part of demonstrating the above concept. For example, a relationship between the $\mathrm{x}$-axis vibration, temperature and surface roughness average variable (e.g. Ra) is identified. More specifically, with an increase in vibration and temperature, an increase in $R a$ is detected which triggers a lower surface quality of the machined parts and is a clear indicator of progressing tool-wear, which if not addressed, will eventually lead to breakage. The correlation between the three mentioned variables is performed for all of the experiments, which can be seen in Figures 9 to 14.

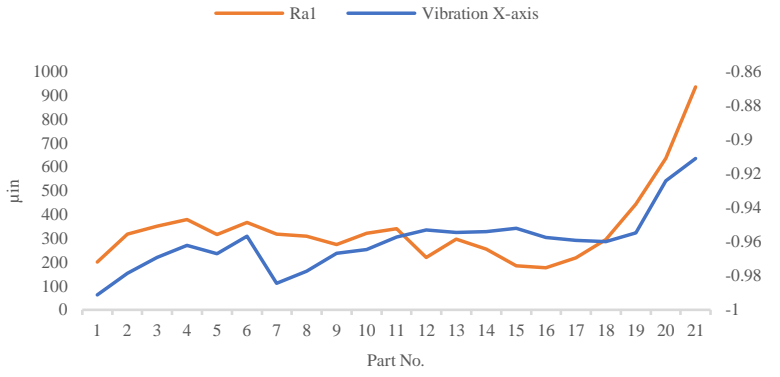

Fig. 9. Vibration X-Axis vs. Roughness Average

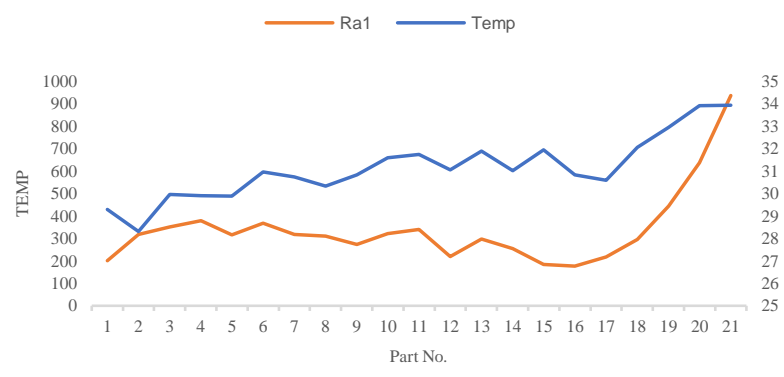

Fig.10. Temperature vs. Roughness Average

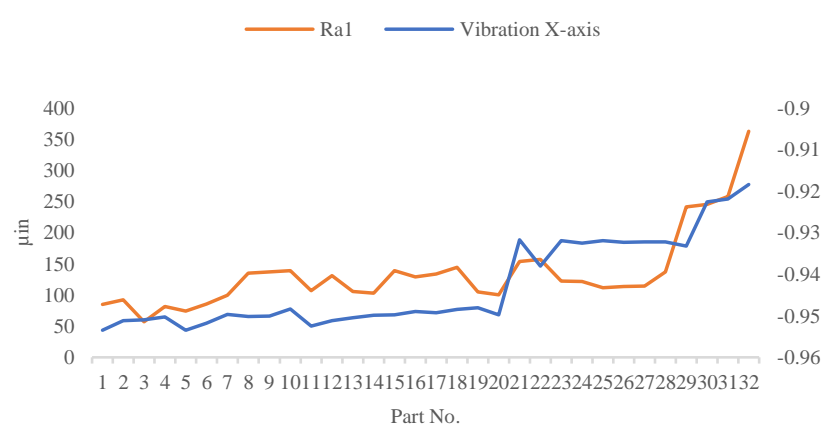

Fig. 11. Vibration X-Axis vs. Roughness Average

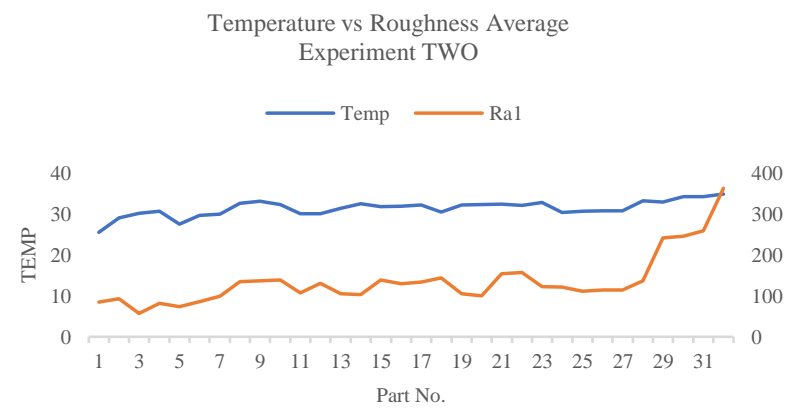

Fig. 12. Temperature vs. Roughness Average

Vibration x-Axis vs. Roughness Average Experiment THREE
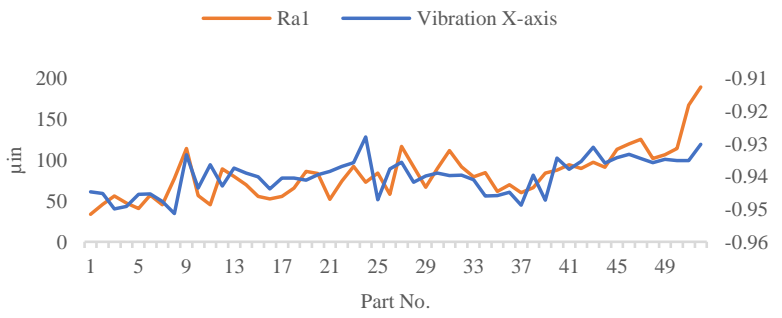

Fig. 13. Vibration X-Axis vs. Roughness Average

Temperature vs. Roughness Average Experiment THREE
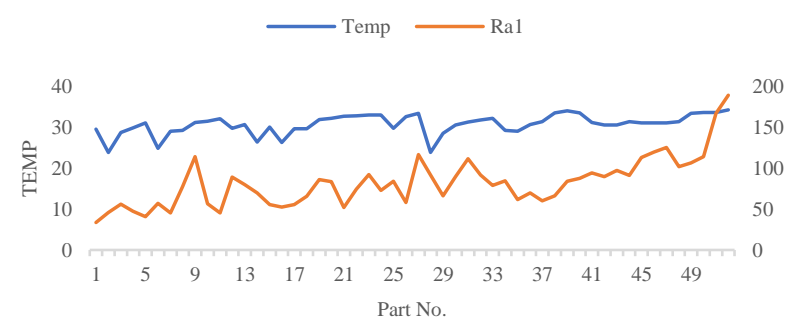

Fig. 14. Temperature vs. Roughness Average

Secondarily, the single-board computer (the Raspberry Pi 3 Model B) and the IIoT device (the Sense-Hat) performed with high durability and stability during the experiments, even though the Raspberry Pi case suffered severe scratches. Thus, proved that they can be used under certain harsh and various machining conditions close to an industrial-grade enclosure. Nevertheless, further research about this matter is recommended. 
Additionally, the embedded processing capability was demonstrated by integrating the Recursive Partitioning and Regression Tree model (rpart), achieving encouraging results. More specifically, the (rpart) model predicts with an average accuracy of $81 \%$ at which processing part the quality threshold will be exceeded. Figure 15 shows the resulting decision tree. As it can be seen in Figure 15, the root node, which is on the topmost is primarily divided by the mean values of temperature and further divided by mean values of $\mathrm{X}$-axis and the standard deviation of temperature values. Consequently, the (rpart) model calculates the variable importance in which the mean value of temperature has an importance of $34 \%$, mean value of $\mathrm{X}$-axis of $31 \%$, standard deviation of temperature of $30 \%$ and standard deviation of $\mathrm{X}$-axis of $6 \%$.

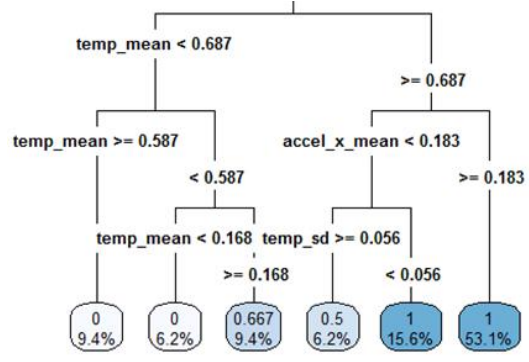

Fig. 15. Recursive Partitioning and Regression Tree

In the above tree, the target variable is a categorical value and the predictor variables are continuous values thus the results are given as probabilities. Table 2 shows the predictions and the actual values for Experiment in which the false predictions are highlighted in red and the correct ones in green.

Table 2. Predictions for Experiment ONE
\begin{tabular}{|c|c|c|}
\hline Part No. & Predictions & Actual Values \\
\hline 1 & 0.6666666667 & 1 \\
\hline 2 & 0.6666666667 & 1 \\
\hline 3 & 0.6666666667 & 1 \\
\hline 4 & 0.6666666667 & 1 \\
\hline 5 & 0.0000000000 & 1 \\
\hline 6 & 0.0000000000 & 1 \\
\hline 7 & 0.50000000000 & 1 \\
\hline 8 & 1 & 1 \\
\hline 9 & 1 & 1 \\
\hline 10 & 1 & 1 \\
\hline 11 & 1 & 1 \\
\hline 12 & 1 & 1 \\
\hline 13 & 1 & 1 \\
\hline 14 & 1 & 1 \\
\hline 15 & 1 & 1 \\
\hline 16 & 0 & 1 \\
\hline 17 & 0 & 1 \\
\hline 18 & 1 & 1 \\
\hline 19 & 1 & 1 \\
\hline 20 & 1 & 1 \\
\hline 21 & 1 & 1 \\
\hline
\end{tabular}

As it can be seen in Table 2, all the actual roughness average variables exceeded the set quality threshold whereas the model predicts 17 values correctly and four values wrongly. Naturally, this is just an indicative result and more advanced experimentation setups and data processing can be employed instead, within the Industry 4.0 -enabled monitoring concept presented in this paper.

The performed predictions for a consequent set of experiments had similar accuracy wherein 42 roughness values are identified correctly and 10 values wrongly. The model shows the negative trend of tool-wear after a certain number of machined parts for the experiments in which preventive actions can be taken in advance before a breakdown is reached. Realtime data can be fed into the model in which the rejection of machined parts with any kind of threshold and machining parameters can be forecasted.

In summary, an increase in the vibrations of $\mathrm{X}$-axis and temperature values affect the roughness average variable negatively in which the model predicts the same negative trend with consecutive 1's. As well, the model is able to predict with an average accuracy of $81 \%$ when a machined part will exceed a certain quality threshold, which can be correlated to the condition of the tool in which the maintenance department can be alerted in advance in order to avoid tool wearing or breakage and to assure constant quality standards during production.

\section{CONCLUSIONS}

Different manufacturing sectors and/or industrial areas are showing a growing interest in the implementation of Industry 4.0 technologies to develop competitive advantage strategies such as predictive maintenance strategies. Industry 4.0 has proven to be a beneficial "vision" for the today's (smart) industries and factories due to its high efficiency, cost reduction, digitalization, connectivity and dynamic decision-making processes nature. New potentials are available from ITs/OTs, such as CPSs, data analytics, as well as ML techniques, leading to introduce new capabilities for PdM systems, serving as powerful "tools" to improve production and maintenance activities, to assure high quality standards, to reduce costs and downtimes, and to gain significant insights about production processes. Nonetheless, many research approaches have shown that the development of PdM systems needs significant improvements in terms of reduction of costs for monitoring equipment and monitoring it with higher accuracy. Therefore, the development of PdM systems can take advantage of the introduction of Industry 4.0 technologies.

In conclusion, this research work presents an Industry 4.0enabled approach focused on predictive maintenance, which provides an automated and low-cost data acquisition and condition monitoring functionality for a turning process. In addition, the production process was simulated to capture the mentioned parameters within the machining process and a quality control of the machined parts was conducted to discover correlations between temperature, vibration and roughness average. While the concept presented in this paper employed a specific hardware setup for embedded data acquisition and computing more advanced features (sensors, embedded boards, cloud infrastructure and computing) can be used to expand the scope in further studies.

\section{REFERENCES}

[1] M. Holgado, M. Macchi, and L. Fumagalli. "Maintenance Business Model: A Concept for Driving Performance Improvement". Int'1. Journal of Strategic Engineering Assets Management, 2(2), pp. 59-176, 2015.

[2] D. N. P. Murthy, A. Atrens, and J. A. Eccleston. "Strategic Maintenance Management". Journal of Quality in Maintenance Engineering, 8(4), pp. 287-305, 2002.

[3] U. Al-Turki. "A Framework for Strategic Planning in Maintenance". Journal of Quality in. Maintenance Engineering, 17(2), pp. 150-162, 2011.

[4] A. H. C. Tsang. "Strategic Dimensions of Maintenance Management". Journal of Quality in. Maintenance Engineering, 8(1), pp. 7-39, 2002. 
[5] G. Waeyenbergh, and L. Pintelon. "A Framework for Maintenance Concept Development". Journal of Production Economics, 77(3), pp. 299-313, 2002.

[6] J.M. Simoes, C. F. Gomes, and M. M. Yasin, "A Literature Review of Maintenance Performance Measurement: Directions for Future Research". EnAN-PAD, pp. 1-15, 2010.

[7] R. Dekker. "Applications of Maintenance Optimization Models: A Review and Analysis”. Reliability Engineering \& System Safety, 51(3), pp. 229-240, 1996.

[8] M. Macchi, A. Crespo-Márquez, M. Holgado, L. Fumagalli, and L. Barberá-Martínez. "Value-driven Engineering of E-maintenance Platforms". Journal of Manufacturing Technology Management, 25(4), pp. 568-598, 2014.

[9] T. Han and B.S. Yang. "Development of an e-Maintenance System Integrating Advanced Techniques". Computers in Industry, 57(6), pp. 569-580, 2006.

[10] J. Campos. "Current and Prospective Information and Communication Technologies for the e-Maintenance Applications". Journal of Quality in Maintenance Engineering, 20(3), pp. 233-248, 2014.

[11] J. Lee, J. Ni, D. Djurdjanovic, H. Qiu, and H. Liao. "Intelligent Prognostics Tools and e-Maintenance". Computers in Industry, 57(6), pp. 476-489, 2006.

[12] M. Sameer, K. Muztoba, D. Romero, and T. Wuest. "Smart Manufacturing: Characteristics, Technologies and Enabling Factors". Proceedings of the Institution of Mechanical Engineers, Part B: Journal of Engineering Manufacture, DOI: 10.1177/0954405417736547.

[13] A. Crespo Marquez, B. Iung, and E. Levrat. "On the Concept of e-maintenance: Review and Current Research". Reliability Engineering \& System Safety, 93(8), pp. 1165-1187, 2008.

[14] B. Iung, E. Levrat, A.C. Marquez, and H. Erbe. "Conceptual Framework for e-Maintenance: Illustration by e-Maintenance Technologies and Platforms". Annual Reviews in Control, 33(2), pp. 220-229, 2009.

[15] A. Voisin, E. Levrat, P. Cocheteux, and B. Iung. "Generic Prognosis Model for Proactive Maintenance Decision Support: Application to PreIndustrial e-Maintenance Testbed". Journal of Intelligent Manufacturing, 21(2), pp. 177193, 2010

[16] H.R. Golmakani. "Optimal Age-based Inspection Scheme for ConditionBased Maintenance using A* Search Algorithm". Journal of Production Research, 50(23), pp. 7068-7080, 2012.

[17] J. Koochaki, J.A.C. Bokhorst, H. Wortmann, and W. Klingenberg. "The Influence of Condition-Based Maintenance on Workforce Planning and Maintenance Scheduling". Journal of Production Research, 51(8), pp. 113, 2012.

[18] Q. Zhu, H. Peng, and G.J. van Houtum. “A Condition-Based Maintenance Policy for Multi-Component Systems with a High Maintenance Setup Cost”. OR Spectrum, 37(4), pp. 1007-1035, 2015.

[19] J. Koochaki, J.A.C. Bokhorst, H. Wortmann, and W. Klingenberg, "CBM in the Context of Opportunistic Maintenance". Journal of Production Research, 50(23), pp. 6918-6929, 2012.

[20] R.C.M. Yam, P.W. Tse, L. Li, and P. Tu. "Intelligent Predictive Decision Support System for Condition-Based Maintenance". Int'l. Journal of Advanced Manufacturing Technology, 17(5), pp. 383-391, 2001.

[21] Levrat, E., Iung, B. and Crespo Marquez, A. "E-maintenance: Review and Conceptual Framework”. Production Planning \& Control, 19(4), pp. 408429, 2008.

[22] M.M. Tehrani, Y. Beauregard, M. Rioux, J.P. Kenne, and R. Ouellet. “A Predictive Preference Model for Maintenance of a Heating Ventilating and Air Conditioning System". IFAC Proceedings, 48(3), pp. 130-135, 2015

[23] C. Park, D. Moon, N. Do, and S.M. Bae. "A Predictive Maintenance Approach based on Real-time Internal Parameter Monitoring". Int'l. Journal of Advanced Manufacturing Technology, 85(1-4), pp. 623-632, 2015.

[24] M. Carnero. "An Evaluation System of the Setting up of Predictive Maintenance Programmes". Reliability Engineering \& System Safety, 91(8), pp. 945-963, 2006.

[25] G.A. Susto, A. Schirru, S. Pampuri, S. McLoone, and A. Beghi. "Machine Learning for Predictive Maintenance: A Multiple Classifier Approach". IEEE Transactions on Industrial Informatics, 11(3), pp. 812-820, 2015.

[26] R. Baidya, and S.K. Ghosh. "Model for a Predictive Maintenance System Effectiveness Using the Analytical Hierarchy Process as Analytical Tool”. IFAC-PapersOnLine, 48(3), pp. 1463-1468, 2015
[27] A. Nadgir. "Prediction of Flank Wear by Using Back Propagation Neural Network Modeling when Cutting Hardened H-13 Steel with Chamfered and Honed CBN Tools". Int'1. Journal of Machine Tools and Manufacture 42(2):287-297, 2002.

[28] S. Weyer, M. Schmitt, M. Ohmer, D. Gorecky. "Towards Standardization as the Crucial Challenge for Highly Production Systems for Highly Modular, Multi-vendor Production Systems". IFAC-PapersOnLine, 48(3), pp. 579-584, 2015

[29] K. Bob. "Industrial Internet of Things (IIoT) Benefits, Examples". 2015 http://www.controleng.com/single-article/industrial-internet-of-thingsiiot-benefits-examples/a2fdb5aced1d779991d91ec3066cff40.html.

[30] J. Lee, H.D. Ardakani, S. Yang, and B. Bagheri. "Industrial Big Data Analytics and Cyber-Physical Systems for Future Maintenance \& Service Innovation". Procedia CIRP, 38, pp. 3-7, 2015.

[31] Dothang Truong. "How Cloud Computing Enhances Competitive Advantages: A Research Model for Small Businesses”. The Business Review, Cambridge 15(1), pp.59-65, 2010.

[32] G.A. Susto, J. Wan, S. Pampuri, M. Zanon, A.B. Johnston, et al. “An Adaptive Machine Learning Decision System for Flexible Predictive Maintenance”. Automation Science \& Engineering, pp. 806-811, 2014.

[33] S. Landset, T.M. Khoshgoftaar, A.N. Richter, and T. Hasanin. "A Survey of Open-Source Tools for Machine Learning with Big Data in the Hadoop Ecosystem". Journal of Big Data, pp. 1-36, 2015.

[34] T. Xia, L. Xi, X. Zhou, and J. Lee. "Condition-Based Maintenance for Intelligent Monitored Series System with Independent Machine Failure Modes". Int'l. Journal of Production Research, 51(15), pp. 4585-4596, 2013.

[35] A.K.S. Jardine, D. Lin, and D. Banjevic. "A Review on Machinery Diagnostics and Prognostics Implementing CBM". Mechanical Systems and Signal Processing, 20(7), pp. 1483-1510, 2006.

[36] H.M. Hashemian, S. Member, W.C. Bean, and S. Member. "State-of-theArt Predictive Maintenance Techniques". 60(10), pp. 3480-3492, 2011.

[37] R. Huang, L. Xi, J. Lee, and C.R. Liu. "The Framework, Impact and Commercial Prospects of a New Predictive Maintenance System: Intelligent Maintenance System”. Production Planning and Control, 16(7), pp. 652-664, 2005 
2018-08-16

\section{An industry 4.0-enabled low cost} predictive maintenance approach for SMEs: a use case applied to a CNC

\section{turning centre}

Sezer, Erim

IEEE

Erim Sezer, David Romero, Federico Guedea, et al., An industry 4.0-enabled low cost predictive maintenance approach for SMEs: a use case applied to a CNC turning centre. 2018 IEEE International Conference on Engineering, Technology and Innovation (ICE/ITMC), 17-20 June 2018, Stuttgart, Germany https://doi.org/ 10.1109/ICE.2018.8436307

Downloaded from Cranfield Library Services E-Repository 